\title{
Potential Force Algorithm with Kinematic Control as Path Planning for Disinfection Robot
}

\author{
Iswanto Suwarno ${ }^{1 *}$, Wiwin A. Oktaviani ${ }^{2}$, Yosi Apriani ${ }^{3}$, Dhiya Uddin Rijalusalam ${ }^{4}$, Anish Pandey ${ }^{5}$ \\ ${ }^{1}$ Department of Engineer Professional Program, Universitas Muhammadiyah Yogyakarta, Yogjakarta, Indonesia \\ 1,4 Department of Electrical Engineering, Universitas Muhammadiyah Yogyakarta, Yogyakarta, Indonesia \\ ${ }^{2,3}$ Department of Electrical Engineering, Universitas Muhammadiyah Palembang, Palembang, Indonesia \\ ${ }^{1,4}$ Center for Robotics and Artificial Intelegent Center (RAIC), Universitas Muhammadiyah Yogyakarta, Yogyakarta, \\ Indonesia \\ 1, 2, 3, 4,5 Kalinga Institute of Industrial Technology (KIIT) Deemed to be University, An Institute of Eminence, Bhubaneswar- \\ 751024, India \\ Email: ${ }^{1}$ iswanto_te@umy.ac.id, ${ }^{2}$ wiwin_oktaviani@um-palembang.ac.id, ${ }^{3}$ yosi_apriani@um-palembang.ac.id, \\ ${ }^{4}$ dhiya.uddin.ft18@mail.umy.ac.id, ${ }^{5}$ anish06353@gmail.com \\ *Corresponding Author
}

\begin{abstract}
The disinfection robot is a virus-sterilizing robot that uses a nonholonomic robot model. Route planning algorithms are needed to allow disinfection robots to sterilize rooms in unknown areas and perform the task while navigating using a potential field algorithm. There is a problem applying the algorithm to nonholonomic robots: avoiding obstacles. The proposed route planning algorithm has been transformed into a potential force used to plan the path of disinfection robots in static and dynamic environments and environments with static obstacles. A potential field algorithm is used. There are some issues when the potential force algorithm is applied to nonholonomic disinfection robots in the area. Like any other robot, it takes a long time to avoid static obstacles. Therefore, this paper proposed a potential force algorithm that allows a robot to move towards a target point while avoiding static obstacles. The algorithm showed that a modified potential field algorithm with potential force could be applied to differentialdriven robots for path planning. The disinfection robot could avoid obstacles with a faster response using this algorithm.
\end{abstract}

Keywords-non-holonomic; static obstacles; disinfection robot; path planning; modified potential field

\section{INTRODUCTION}

The Covid-19 pandemic, which started in early 2020, has revolutionized human life and everyday life, later known as the New Normal. Some researchers have done the research and applied the work to make new normal changes for the Covid-19 pandemic. Covid-19 has made scientific communication an open science paradigm studied by Hayashi [1]. The enhancement of Angkring trader's marketing strategy via social media during the Covid-19 era at Ponolog was carried out by Alam [2]. In addition, some researchers made breakthrough investigations related to Covid-19 effects in various aspects of life. For example, the experience of the Covid-19 pandemic for emergency care with conventional treatment habits was reviewed by Mitchell [3]. A new normal lifestyle in Japan's Covid-19 era regarding the trend of emergency transportation was studied by Uryu [4]. A comprehensive diabetic wound care program at home during the Covid-19 pandemic in Yogyakarta was investigated by Wantonoro [5]. Then, the multimodal cardiovascular imaging was safely updated to new normal behavior within the Covid-
19 pandemic investigated by Zoghbi [6]. Covid-19 and continuation of selective normalization surgeries were investigated by Mendes [7].

Among the many habits that have changed are the habits of sterilizing body parts, equipment, and spaces [8]. Several researchers have researched sterilization, especially for Covid-19 disease. Jiang [9] found that ethanol causes significant changes in the chemical composition of air for making indoor disinfectant sprays in homes. The evaluation of the virus-killing activity of the four disinfectants against SARSCoV2 was investigated by Huang [10]. Sterilization of public spaces has become a new practice in society. Sterilization is designed to prevent public places from spreading the disease. Sterilization is done manually or automatically using a disinfection robot. A disinfection robot is an autonomous robot that moves while carrying a sterilizer filled with disinfectant. Disinfection robots are solutions for sterilizing rooms in buildings and public areas, which usually have relatively large areas such as mosques and prayer rooms.

Robots need a route planning method to sterilize the mosque floor from the starting point to the destination. Previous researchers have studied route planning methods, such as in Multi UAV routing method for deep learning radio data acquisition from Bayerlein [11]. The method of route planning for coordinated autonomous transport robots was investigated by Balatti [12]. Zhang investigated how to plan the course of an unmanned aerial vehicle based on ant habits. [13] How to design a logistics path with four fine parameters of a ring-shaped path was investigated by Upadhyay [14]. A combined strategy for online trajectory optimization of unmanned combat aerial vehicles was investigated by Dong [15]. The evolution method of route planning is Z. It is derived by the quantum annealing Hamilton algorithm investigated by Huang [16]. Analog VLSI was used for multipurpose path planning for configurable autonomous robots and was investigated by Koziol [17]. Voronoi diagrams and computational geometry techniques used to route robots moving in dynamic environments have been investigated by Ayawli [18]. The trailer multi-steering system uses a consistent route planning method studied by 
the original [19]. Path design methods for detecting resource overload under project ambiguity conditions were investigated by Pelican [20]. The hybrid A * algorithm was used for stochastic robust path planning of non-holonomic mobile robots. This was investigated by Schafle [21]. A knowledge-based sample route plan for the automatic parking of vehicles was considered by Dong [22]. The potential fieldbased path planning of an automated broom scan from the hybrid sweep area for 5-axis free-form surface inspection was investigated by Zhang [23]. The route planning scheme for redundant manipulator robots investigated by Guo [24] uses a new pseudo-reciprocal with PID properties. Route planning with parking awareness for mechanical parking systems was considered by Ma [25]. A parallelization algorithm for realtime path shortening of high DOF manipulators was studied by Seo [26]. Meanwhile, Cui [27] investigated the V-shaped Nomex honeycomb robot designed for cutting with a straight knife tool; route planning was used to achieve the goal. $\mathrm{Li}$ [28] used adaptive quantum responsive particle swarm optimization for AUV docking pathway planning method. A neural spiking algorithm with axonal delay for path planning of adaptive robots was investigated by Hwu [29]. Based on Sun's research [30], fuzzy path planning with optimally-safe angular velocity was applied for tracking control of warehouse robots.

In research by Liu [31], Liu integrated a jump A * algorithm with a fusion algorithm using a dynamic window approach to design path planning for global and dynamic use. Based on XU's research, we used a multipurpose particle swarm to optimize the trajectory of a rotating unmanned aerial vehicle in rugged terrain [32]. In research by Yuan [33], a GRURNN network model was considered to plan mobile robots' path dynamically. Meanwhile, adaptive discretization was used for optimal path planning in the various timestreams [34]. An overview of cerebrovascular segmentation and modeling for surgical planning was examined by Ajam [35].

Among all path planning methods, the Artificial Potential Field (APF) algorithm is commonly used to control the coordination of multi-robots so that they do not collide with each other while avoiding obstacles. However, the algorithm has some problems when applied to nonholonomic robots. It is challenging to practically design and implement potential field algorithms to the control system of nonholonomic robots due to their kinematics and dynamics. Of these issues, the studies proposed a novel and different method from the aforementioned studies. The contribution of this paper is to introduce an extensively modified field potential algorithm so that this algorithm can be applied to nonholonomic robots, which disinfection robots belong to this class. In addition to changing the tensile force, this paper changes the repulsive force of the artificial potential field algorithm so that areas with local minima, static and dynamic obstacles can be quickly avoided when planning the path of the nonholonomic disinfection robot.

\section{Formulation OF THE PROBLEM}

Nonholonomic disinfection robots have problems with the combination of kinematic control and routing algorithms. In addition, it is difficult to control the robot's movement from its starting position to the desired position while avoiding static obstacles in an unfamiliar environment. In the potential field algorithm, magnetic fields are commonly used to route planning the robot. However, it has many problems when applied to nonholonomic robots, one of which is the attractive force. When the robot's focus is too big, the attractive force will also become too big. Meanwhile, the repulsive force does not work optimally in nonholonomic robots. Therefore, the potential field algorithm must be modified to be applied in nonholonomic robots. It is assumed that the robot's shape, position, velocity, target point position, and polygonal static obstacles are known to simplify the analysis using the artificial potential field algorithm.

\section{MATHEMATICS MODELING FROM DiFFERENTIAL MOBILE DRIVE RoBOT}

The disinfection robot belongs to the class of nonholonomic robots. Before an algorithm can control the robot, the robot must first be modeled. Non-holonomic robots are a type of differentially driven mobile robots. The mobile differentially-driven mobile robot (DDMR) is a type of mobile robot [36]-[39], and its movement is caused by the speed difference between the left and right wheels, as shown in Fig. 1. The figure shows that the robot moves only on the $x-y$ axis.

The proposed model configuration of the disinfection robot basically had two main wheels, each driven by a separate drive (usually a permanent magnetic DC motor with a reducer used to amplify the motor torque). The robot was also equipped with one or two swivel casters. The rear part was attached to the robot as a counterweight [40]-[43].

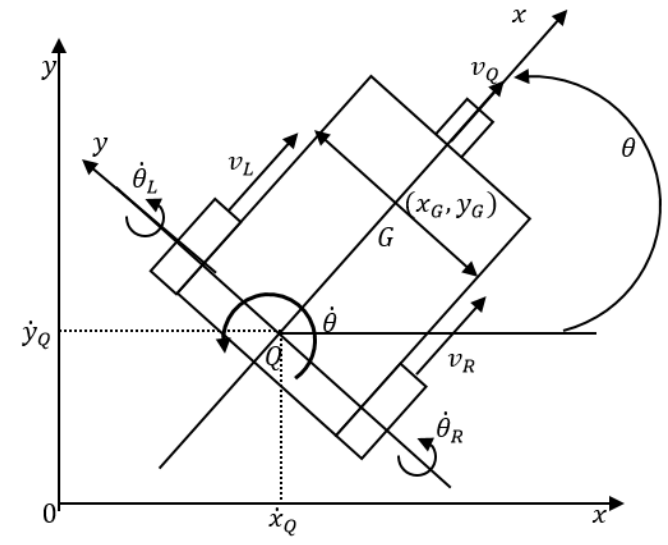

Fig. 1. DDMR on a 2D Cartesian plane

The robot was modeled as a rigid body on wheels in a 2D plane. Fig. 1 also shows that the points $\dot{x}_{Q}$ and $\dot{y}_{Q}$ are the connection points between the coordinates of the robot body $x$ and $y$ and the center of the robot $Q, v_{Q}$ is the linear velocity of the DDMR robot, $v_{L}$ is the linear velocity of the left wheel, $v_{R}$ is the linear velocity of the right wheel, $Q$ is the center of the robot, and $G$ is the center of gravity [44]-[47]. By ignoring the distance between the joints and the degree of freedom between robot and its wheels, the configuration of a mobile robot can be described as three general variables $q(t)$ that describe the robot's position and control input $u(t)$. This can be defined in the matrix below [48]-[50]. 


$$
\begin{gathered}
q(t)=(x(t), y(t), \theta(t))^{T}, \\
u(t)=\left(\dot{\theta}_{R}(t), \dot{\theta}_{L}(t)\right)^{T} .
\end{gathered}
$$

Thus, the linear velocity of the robot can be expressed as equation (3), while the angular velocity can be expressed as equation (2). Each equation consists of the respective equation for left and right wheels.

$$
\begin{aligned}
& \dot{\theta}_{R}(t)=\frac{1}{2 \pi r} v_{R}(t), \\
& \dot{\theta}_{L}(t)=\frac{1}{2 \pi r} v_{L}(t), \\
& v_{R}(t)=v(t)+b \dot{\theta}(t), \\
& v_{L}(t)=v(t)-b \dot{\theta}(t) .
\end{aligned}
$$

Equations above were used to find the linear equation in (4), which can be derived to be the equation for the robot's angular velocity,

$$
\begin{gathered}
v_{Q}(t)=\frac{1}{2}\left(v_{R}(t)+v_{L}(t)\right), \\
\dot{\theta}(t)=\frac{v_{R}(t)-v_{L}(t)}{2 b} .
\end{gathered}
$$

where $v_{Q}(t)$ is the robot's linear velocity variable and $\dot{\theta}(t)$ is the robot's angular velocity.

Movement restriction is one of the problems in DDMR, which is also commonly called non-holonomic constraints. They were made of two main assumptions of the robot. The first assumption is that the robot does not skid, which means there will be no orthogonal slide to the wheel plane. Then, the robots are supposed only to move back and forth but cannot move sideways. Therefore, the wheel velocity equation can be obtained by adding the $\mathrm{x}$-axis and $\mathrm{y}$-axis linear velocities, as shown in Fig. 2. This figure shows the linear velocity equation for the wheel, which can be explained as follows:

$$
v(t)=\dot{x}(t) \cos \theta(t)+\dot{y}(t) \sin \theta(t) .
$$

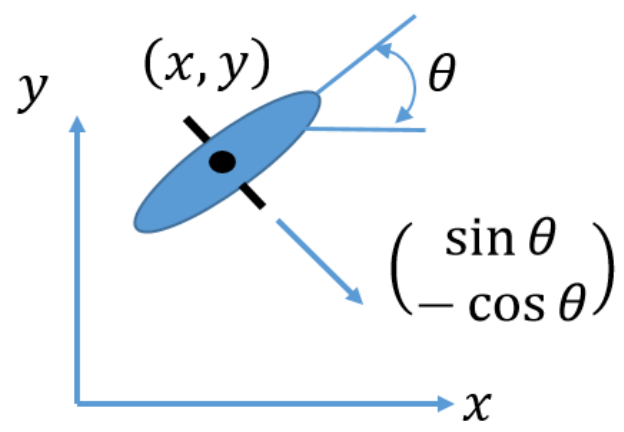

Fig. 2. No lateral slip
The equation for the velocity of the wheel at center $G$ along the horizontal axis is zero, and as shown in (6), the wheel moves only along the $x$-axis and $y$-axis.

$$
0=\dot{x}(t) \cos \theta(t)+\dot{y}(t) \sin \theta(t)
$$

Assuming that the robot is not sliding on the floor, the wheel has a roll-only restriction. The roll-only restriction can be described as shown in Fig. 3. This figure shows that the linear velocity of the wheel is the product of the wheel radius and the wheel angle. Equation (3) shows the rate equation for the left and right wheel speeds,

$$
\begin{aligned}
& v_{R}(t)=r \dot{\theta}_{R}, \\
& v_{L}(t)=r \dot{\theta}_{L} .
\end{aligned}
$$

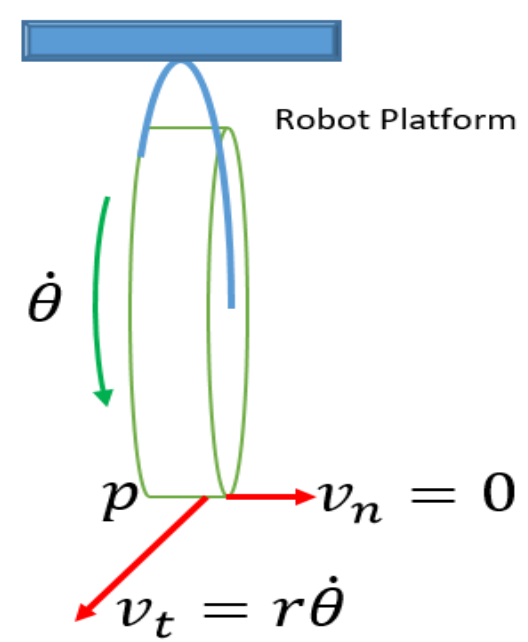

Fig. 3. Pure rolling motion limitation

where $v(t)$ is the linear velocity of the rod, $r$ is the radius of the wheel, and $\theta$ is the angular position. The equations for the position of the robot with wheels are Cartesian fields $x y$, $x(t)=v(t) \sin \theta(t), \quad y(t)=v(t) \cos \theta(t), \quad$ and $\theta(t)=$ $v_{\theta}(t)$. Therefore, the formula of the transformation matrix can be expressed from the initial position of formula (8).

$$
T_{N H}(q)=\left[\begin{array}{cc}
\frac{r}{2} \cos \theta(t) & \frac{r}{2} \cos \theta(t) \\
\frac{r}{2} \sin \theta(t) & \frac{r}{2} \sin \theta(t) \\
\frac{r}{2 d} & -\frac{r}{2 d}
\end{array}\right]
$$

In addition, the angular velocity $u(t)$ has three parameters as inputs to the robot. That is, $u_{1}(t)=\dot{\theta}_{R}(t), u_{2}(t)=\dot{\theta}_{L}(t)$, and $\dot{\theta}(t)=\omega(t)$. From the three parameters of equation (8) and input equation, the equation of motion model of DDMR is $\dot{q}(t)=T N H(q) u(t)$ or

$$
\left[\begin{array}{c}
\dot{x}(t) \\
\dot{y}(t) \\
\dot{\theta}(t)
\end{array}\right]=\left[\begin{array}{cc}
\cos \theta(t) & 0 \\
\sin \theta(t) & 0 \\
0 & 1
\end{array}\right]\left[\begin{array}{l}
v(t) \\
\omega(t)
\end{array}\right] .
$$




\section{ARtificial Potential FiEld Algorithm}

The Artificial Potential Field (APF) algorithm [51]-[54] was first discovered by Khatib [55], which employs the theory of attractive and repulsive forces such as magnetic fields. In other words, artificial potential field force [56] $F_{A P F}$ is the sum of the gravitational potential field force [57] $F_{a t t}$ and the repulsive potential field force [58] $F_{\text {rep }}$. Equation (10) shows the formula of APF.

$$
F_{A P F}=F_{a t t}+F_{r e p} .
$$

The APF algorithm can be used to control nonholonomic robots by inputting linear velocity equations into kinematic robots [57] [59]. The APF force should be converted to the desired rate equation of APF attractive force $\vec{V}_{G}^{\text {att }}$ using equation (11) [60].

$$
\vec{V}_{G}^{a t t}(x, y)=-\nabla U_{a t t}(x, y),
$$

Equation (11) is a potentially attractive equation used in the attractive reaction rate equation. Potentially attractive equations are partially derived from the $x$, y axes shown in (12) and (13) [51], [61], [62],

$$
\begin{aligned}
& \nabla_{x} U_{a t t}(x, y)=\frac{\partial U_{a t t}(x, y)}{\partial x}, \\
& \nabla_{y} U_{a t t}(x, y)=\frac{\partial U_{a t t}(x, y)}{\partial y},
\end{aligned}
$$

Use the potential gravity equation to get the desired velocity equation for the repulsive force $\rightarrow_{O}^{r e p}$. This is shown in (14) [53], [63], [64].

$$
\vec{V}_{o}^{r e p}(x, y)=\nabla U_{r e p}(x, y)
$$

Equation (14) is the equation for the repulsion potential used in the equation for the repulsion rate. The repulsive potential equation is partially derived around the $x$ and $y$ axes shown in (15) and (16) [65], [66].

$$
\begin{aligned}
& \nabla_{x} U_{r e p}(x, y)=\frac{\partial U_{r e p}(x, y)}{\partial x}, \\
& \nabla_{y} U_{r e p}(x, y)=\frac{\partial U_{r e p}(x, y)}{\partial y},
\end{aligned}
$$

The equation for the potential attractive $U_{a t t}(x, y)$ is shown in (17) and the repulsive potential $U_{\text {rep }}(x, y)$ is shown in (18),

$$
\begin{gathered}
U_{a t t}=\frac{1}{2} k_{a}\left(\left(x_{b}-x_{r e f}\right)^{2}+\left(y_{b}-y\right)^{2}\right), \\
U_{\text {rep }}(x, y)=\left\{\begin{array}{cc}
\frac{1}{2} k_{r}\left(\frac{1}{\rho_{o}}-\frac{1}{r_{o}}\right)^{2} & \text { if } \rho_{o} \leq r_{o}, \\
0 & \text { if } \rho_{o}>r_{o}
\end{array}\right.
\end{gathered}
$$

where $k_{a}$ is the potential constant of attraction, $\left(x_{b}, y_{b}\right)$ is the position of the robot, $\left(x_{r e f}, y_{r e f}\right)$ is the position of the target point, $k_{r}$ is the potential constant of repulsion, and $r_{o}$ is the distance limitation from the influence of repulsion potential. Meanwhile, $\rho_{o}$ is the shortest distance between the robot and the obstacle, and can be calculated using the following equation [67] [68]:

$$
\rho_{o}=\sqrt{x_{o r}^{2}-y_{o r}^{2}} \text {, }
$$

where $x_{o r}$ is the difference in distance between the robot on the $x$-axis and the obstacle, and $y_{\text {or }}$ is the difference in distance between the robot on the $y$-axis and the obstacle. Desirable velocity equation of attractive force APF force $\vec{V}_{G}^{a t t} x, y$-axis is expressed as follows [62]:

$$
\begin{aligned}
& \vec{V}_{G_{x}}^{a t t}=-k_{a}\left(x_{b}-x_{\text {ref }}\right), \\
& \vec{V}_{G_{y}}^{a t t}=-k_{a}\left(y_{b}-y_{\text {ref }}\right),
\end{aligned}
$$

Meanwhile, desirable velocity equation of repulsive force $\mathrm{APF} \underset{V_{O}}{r e p} x, y$-axis is written as follows.

$$
\begin{gathered}
\vec{V}_{O_{x}}^{r e p}=\left\{\begin{array}{cc}
-k_{r}\left(1-\left(\frac{\rho_{o}}{r_{o}}\right)\right)\left(\left(\frac{x_{o r}}{\rho_{o} r_{o}}\right)\right) & \text { if } \rho_{o} \leq r_{o}, \\
0 & \text { if } \rho_{o}>r_{o}
\end{array},\right. \\
\vec{V}_{o_{y}}^{r e p}=\left\{\begin{array}{cl}
-k_{r}\left(1-\left(\frac{\rho_{o}}{r_{o}}\right)\right)\left(\left(\frac{y_{o r}}{\rho_{o} r_{o}}\right)\right) & \text { if } \rho_{o} \leq r_{o} \\
0 & \text { if } \rho_{o}>r_{o}
\end{array},\right.
\end{gathered}
$$

Therefore, the formula for the $x$-axis and $y$-axis velocities of the APF force is:

$$
\begin{aligned}
& \vec{V}_{x}^{a p f}=\vec{V}_{G_{x}}^{a t t}+\vec{V}_{o_{x}}^{r e p}, \\
& \vec{V}_{y}^{a p f}=\vec{V}_{G_{y}}^{a t t}+\vec{V}_{o_{y}}^{r e p},
\end{aligned}
$$

\section{PROPOSED CONTROL}

One of the research goals in the paper is to apply APF force to the disinfection robot categorized as a nonholonomic robot. It can be overcome by applying mathematical substitutions and taking several considerations. The velocity equations on the $\mathrm{x}$ and $\mathrm{y}$ axes of the APF force in (25) can be substituted into the non-holonomic modeling equation in equation (9). In the study, the robot is supposed to be a mass point. Therefore, the robot's position in absolute coordinates and its direction must also be known. Then, there are limitations in non-holonomic robots, commonly regarded as non-holonomic constraints. They can be overcome using kinematic control as expressed in (9) with APF as the path reference.

The system starts with the APF algorithm, whose input data comes from the environment shown in Fig. 4. It consists 
of an obstacle, starting position, and destination. Using APF, path mapping is produced for the robot's kinematic control to avoid obstacles while navigating to the targeted position. Moreover, APF also plays an essential role in the overall control system. Since the outputs of APF are used for position and orientation references, APF also takes part in determining whether the control system should be stopped or re-iterated, calculating the next destination point.

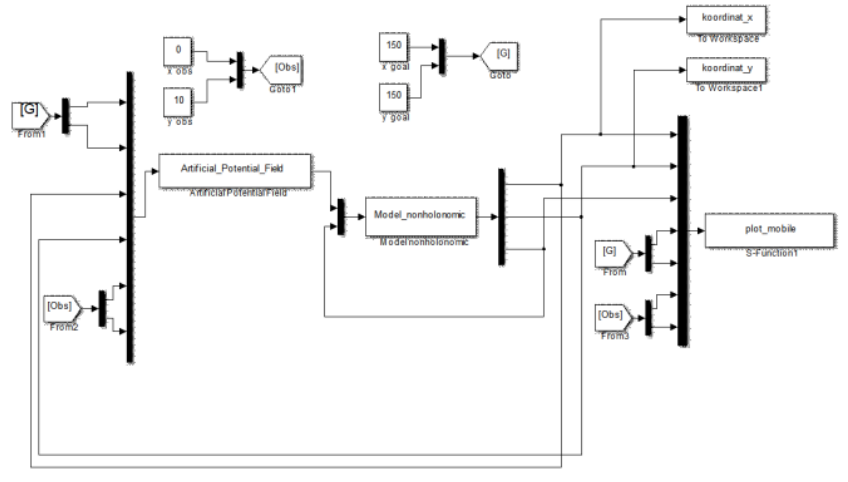

Fig. 4. System process

\section{Modified ARTIFICIAL Potential Field ALGORITHM}

The problem in this research is to apply the APF algorithm to non-holonomic robots. The APF algorithm is modified in its attractive force as shown before in equations (20) (21) and repulsive as in equations (22) (23). The desired velocity equation for the modification of the attractive force APF $\vec{V}_{G}^{\text {att }}$ on the $x, y$ axis is written as follows

$$
\begin{aligned}
& \vec{V}_{G_{x}}^{a t t}=k_{a}\left(x_{b}-x_{\text {ref }}\right), \\
& \vec{V}_{G y}^{a t t}=k_{a}\left(y_{b}-y_{\text {ref }}\right),
\end{aligned}
$$

From the equation, it can be seen that the velocity of the APF Attractive force $\rightarrow_{V}^{\text {att }}$ on the $x, y$ axis is modified by making the destination point a positive pole. The desired velocity equation for the modification of the APF Repulsive force $\underset{V_{O}}{r e p}$ on the $x, y$ axis is as follows

$$
\begin{aligned}
& \vec{V}_{o_{x}}^{r e p}=\left\{\begin{array}{cc}
-k_{r}\left(\left(\frac{x_{o r}}{\left(\sqrt{\left(x_{o r}^{2}+y_{o r}^{2}\right)}\right)^{3}}\right)\right) & \text { if } \rho_{o} \leq r_{o}, \\
0 & \text { if } \rho_{o}>r_{o}
\end{array}\right. \\
& \vec{V}_{o_{y}}^{r e p}=\left\{\begin{array}{cl}
-k_{r}\left(\left(\frac{y_{o r}}{\left(\sqrt{\left({x_{o r}}^{2}+y_{o r}^{2}\right)}\right)^{3}}\right)\right) & \text { if } \rho_{o} \leq r_{o}, \\
0 & \text { if } \rho_{o}>r_{o}
\end{array}\right.
\end{aligned}
$$

\section{RESULT AND DisCUSSION}

In this experiment, Khatib's artificial potential field algorithm was tested on a non-holonomic disinfection robot using Matlab software. Matlab was used to design the Khatib [66] artificial potential field algorithm and model the non- holonomic robot. Both algorithms (modified and original by Khatib) were simulated. Then, the performance results of non-holonomic robots reaching their targets and avoiding static obstacles in an unknown environmental model were evaluated. There were several tests conducted on the potential field algorithms.

In the first test, the modified Khatib's artificial potential field algorithm was tested in an unknown environment with no static or dynamic obstacles, as shown in Fig. 5. This figure shows that four experiments were performed at four different targets of the non-holonomic disinfection robot: point A (-80, $-80)$ as shown in the red color curve, $\mathrm{B}(80,80)$ as shown in the blue color, $\mathrm{C}(80,80)$ as displayed in the green curve, and $\mathrm{D}(-80,80)$ as displayed in the pink curve. The robot was placed at the starting point $(0,0)$ and in a flat position at an angle of $270^{\circ}$. The trajectory results of Tracking-1, Tracking3 , and Tracking-4 curves show that the robot rotated first to align with the target point. Meanwhile, the result of tracking2 in the green-colored curve shows that the robot did not rotate since the robot's alignment position had corresponded to the target point.

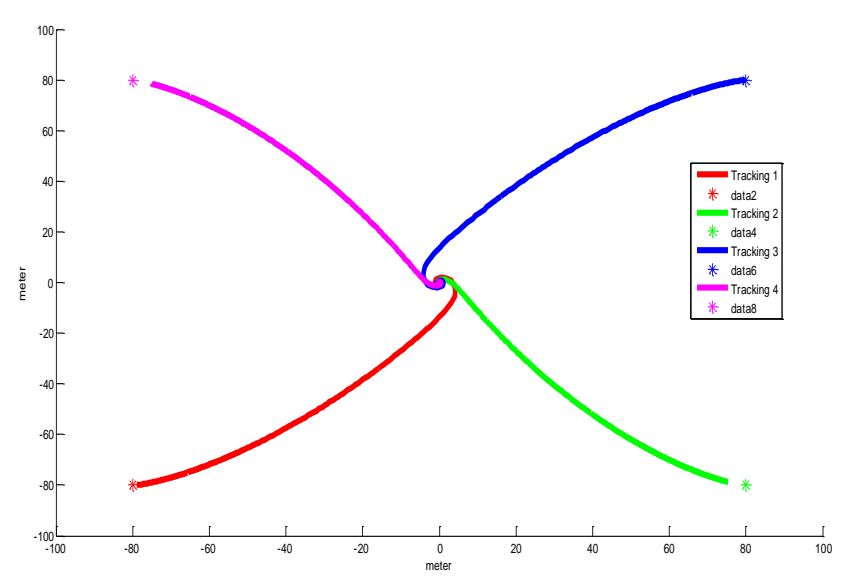

Fig. 5. First test

The modified Khatib's artificial potential field algorithm was tested in an unknown environment with static obstacles in the second test. Four experiments were performed on four different targets of a nonholonomic disinfection robot. The target points and starting position of the robot used for this test were the same as the previous one. However, static obstacles were added in this test's environment. The test results are shown in Fig. 6. Tracking-1, shown in the red curve, indicates that the robot rotated first to provide the alignment position corresponding to the target point. The robot drove to the target point and avoided obstacles at (-20,30). Tracking-2, shown in the green curve, shows that the robot did not rotate because the robot's alignment position corresponds to the target point. The robot drove to the target point and avoided obstacles at $(20,-30)$. Tracking-3, as shown in the blue curve, shows that the robot rotated first to provide the alignment position corresponding to the target point. The robot drove to the target point and avoided obstacles at $(20,30)$. Tracking-4, with a pink curve, shows that the robot rotated first to provide the alignment position corresponding to the target point. The robot drove to the target point and avoided obstacles $(-20,30)$. 


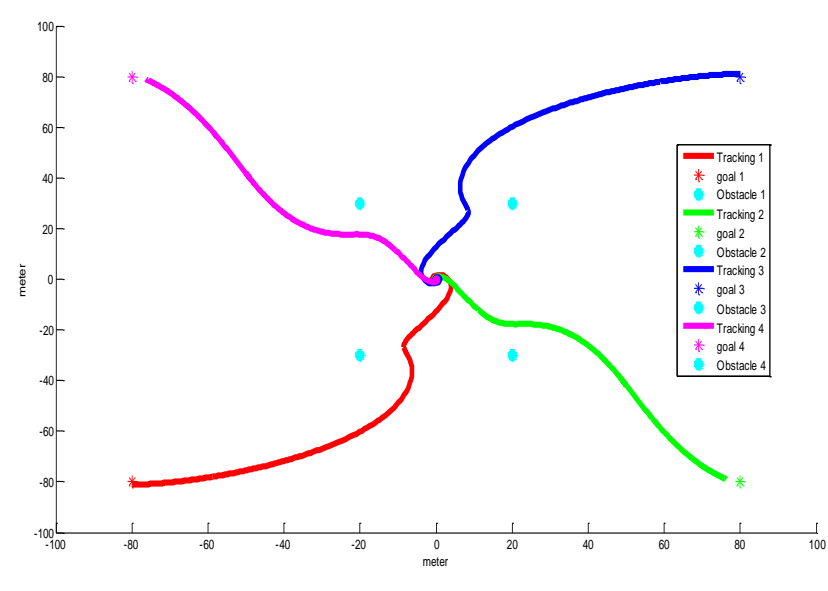

Fig. 6. Second Test

\section{CONCLUSION}

The paper successfully modified Khatib's artificial potential field algorithm to be applied to nonholonomic disinfection robots. Prior to modification, implementing an artificial potential field algorithm to non-holonomic robots on simulation with a far distance between the initial and target position makes the system error because the force exerted on the robot is relatively big. Therefore, the modification was made to allow the robot to reach the target point with less force generated by the algorithm. Other issues related to Khatib's artificial potential field algorithm were also solved in the paper. The algorithm's attractive force and repulsive force affected the robot's stability while used to avoid static and dynamic obstacles. Hence, the proposed modified APF algorithm allow non-holonomic disinfection robot to avoid static obstacles faster than the conventional algorithm made by Khatib.

\section{ACKNOWLEDGMENT}

This research was supported by the Risetmu Project grant from the Diktilitbang Muhammadiyah Central Leadership through the Research Directorate of Universitas Muhammadiyah Palembang with contract number: 0784/I.3/D/2021 dated July 24, 2021, given to A/P Wiwin A. Oktaviani., ST, M. Eng.

\section{REFERENCES}

[1] K. Hayashi, "How Could COVID-19 Change Scholarly Communication to a New Normal in the Open Science Paradigm?," Patterns, vol. 2, no. 1, p. 100191, Jan. 2021.

[2] A. Alam, A. Firdaus, E. Muafiah, M. Heriyudanta, and I. Al, "Empowerment of Marketing Strategies of Angkringan Traders Through Social Media During Covid-19 Time in Ponorogo," J. Pengabdi. dan Pemberdaya. Masy. Indones., vol. 1, no. 3, pp. 84-94, 2021.

[3] R. Mitchell and G. Nou, "A 'new normal': Harnessing the experience of COVID-19 for sustained improvements in global emergency care," Lancet Reg. Heal. - West. Pacific, vol. 1, p. 100012, Aug. 2020.

[4] S. Uryu, Y. Tanoue, S. Nomura, K. Matsuura, K. Makiyama, T. Kawashima, D. Yoneoka, A. Eguchi, Y. Kawamura, S. Gilmour, H. Sakamoto, K. Shimizu, C. F. S. Ng, and M. Hashizume, "Trends in emergency transportation due to heat illness under the new normal lifestyle in the COVID-19 era, in Japan and 47 prefectures," Sci. Total Environ., vol. 768, no. January, p. 144723, May 2021.

[5] Wantonoro, D. Prihatiningsih, and E. Koni, "Comprehensive home based diabetic wounds care program during covid-19 pandemic in
Yogyakarta,” J. Pengabdi. dan Pemberdaya. Masy. Indones., vol. 1, no. 6, pp. 235-240, 2021.

[6] W. A. Zoghbi, M. F. DiCarli, R. Blankstein, A. D. Choi, V. Dilsizian, F. A. Flachskampf, J. B. Geske, P. A. Grayburn, F. A. Jaffer, R. Y. Kwong, J. A. Leipsic, T. H. Marwick, E. Nagel, K. Nieman, S. V. Raman, M. Salerno, P. P. Sengupta, L. J. Shaw, and Y. S. Chandrashekhar, "Multimodality Cardiovascular Imaging in the Midst of the COVID-19 Pandemic," JACC Cardiovasc. Imaging, vol. 13, no. 7, pp. 1615-1626, Jul. 2020.

[7] F. F. Mendes, "COVID-19 and resuming elective surgery. How do we get back to normal?," Brazilian J. Anesthesiol. (English Ed., vol. 70, no. 5, pp. 455-456, Sep. 2020.

[8] S. Sofia, M. Ariani, and Z. Sa, "Socialization of covid-19 prevention for children at kaye aceh village, southwest aceh regency, aceh province, indonesia," J. Pengabdi. dan Pemberdaya. Masy. Indones., vol. 1, no. 4, pp. 142-148, 2021.

[9] J. Jiang, X. Ding, K. P. Isaacson, A. Tasoglou, H. Huber, A. D. Shah, N. Jung, and B. E. Boor, "Ethanol-based disinfectant sprays drive rapid changes in the chemical composition of indoor air in residential buildings," J. Hazard. Mater. Lett., vol. 2, p. 100042, Nov. 2021.

[10] Y. Huang, S. Xiao, D. Song, and Z. Yuan, "Evaluating the Virucidal Activity of Four Disinfectants Against SARS-CoV-2," Am. J. Infect. Control, Nov. 2021.

[11] H. Bayerlein, M. Theile, M. Caccamo, and D. Gesbert, "Multi-UAV Path Planning for Wireless Data Harvesting With Deep Reinforcement Learning," IEEE Open J. Commun. Soc., vol. 2, no. April, pp. 1171-1187, 2021.

[12] P. Balatti, F. Fusaro, N. Villa, E. Lamon, and A. Ajoudani, "A Collaborative Robotic Approach to Autonomous Pallet Jack Transportation and Positioning," IEEE Access, vol. 8, pp. 142191$142204,2020$.

[13] C. Zhang, C. Hu, J. Feng, Z. Liu, Y. Zhou, and Z. Zhang, "A SelfHeuristic Ant-Based Method for Path Planning of Unmanned Aerial Vehicle in Complex 3-D Space With Dense U-Type Obstacles," IEEE Access, vol. 7, pp. 150775-150791, 2019.

[14] S. Upadhyay and A. Ratnoo, "On Existence and Synthesis of Smooth Four Parameter Logistic Paths Inside Annular Passages," IEEE Robot. Autom. Lett., vol. 3, no. 4, pp. 4375-4382, Oct. 2018.

[15] K. Dong, H. Huang, C. Huang, and Z. Zhang, "Trajectory online optimization for unmanned combat aerial vehicle using combined strategy,” J. Syst. Eng. Electron., vol. 28, no. 5, pp. 963-970, Oct. 2017.

[16] Z. Huang, J. Zhang, M. Wu, X. Li, and Y. Dong, "Evolutionary Method of Sink Node Path Planning Guided by the Hamiltonian of Quantum Annealing Algorithm," IEEE Access, vol. 9, pp. 5346653479,2021

[17] S. Koziol, "Multi-Objective Path Planning for Autonomous Robots Using Reconfigurable Analog VLSI," IEEE Access, vol. 8, pp. 80134-80147, 2020.

[18] B. B. K. Ayawli, X. Mei, M. Shen, A. Y. Appiah, and F. Kyeremeh, "Mobile Robot Path Planning in Dynamic Environment Using Voronoi Diagram and Computation Geometry Technique," IEEE Access, vol. 7, pp. 86026-86040, 2019.

[19] J. Yuan, S. Yang, and J. Cai, "Consistent Path Planning for On-AxleHitching Multisteering Trailer Systems," IEEE Trans. Ind. Electron., vol. 65, no. 12, pp. 9625-9634, Dec. 2018.

[20] M. Pelikan, H. Stikova, and I. Vrana, "Detection of Resource Overload in Conditions of Project Ambiguity," IEEE Trans. Fuzzy Syst., vol. 25, no. 4, pp. 868-877, Aug. 2017.

[21] T. R. Schafle and N. Uchiyama, "Probabilistic Robust Path Planning for Nonholonomic Arbitrary-Shaped Mobile Robots Using a Hybrid A* Algorithm," IEEE Access, vol. 9, pp. 93466-93479, 2021.

[22] Y. Dong, Y. Zhong, and J. Hong, "Knowledge-Biased SamplingBased Path Planning for Automated Vehicles Parking," IEEE Access, vol. 8, pp. 156818-156827, 2020.

[23] Y. Zhang and K. Tang, "Automatic Sweep Scan Path Planning for Five-Axis Free-Form Surface Inspection Based on Hybrid Swept Area Potential Field," IEEE Trans. Autom. Sci. Eng., vol. 16, no. 1, pp. 261-277, Jan. 2019. 
[24] D. Guo, F. Xu, and L. Yan, "New Pseudoinverse-Based Path-Planning Scheme With PID Characteristic for Redundant Robot Manipulators in the Presence of Noise," IEEE Trans. Control Syst. Technol., vol. 26, no. 6, pp. 2008-2019, Nov. 2018

[25] S. Ma, H. Jiang, M. Han, J. Xie, and C. Li, "Research on Automatic Parking Systems Based on Parking Scene Recognition," IEEE Access, vol. 5, pp. 21901-21917, 2017.

[26] J. H. Seo, H. Lee, and K.-D. Kim, “A Parallelization Algorithm for Real-Time Path Shortening of High-DOFs Manipulator," IEEE Access, vol. 9, pp. 123727-123741, 2021.

[27] R. Cui, J. Zhang, P. Feng, D. Yu, and Z. Wu, “A Path Planning Method for V-Shaped Robotic Cutting of Nomex Honeycomb by Straight Blade Tool," IEEE Access, vol. 8, pp. 162763-162774, 2020.

[28] Z. Li, W. Liu, L.-E. Gao, L. Li, and F. Zhang, "Path Planning Method for AUV Docking Based on Adaptive Quantum-Behaved Particle Swarm Optimization,” IEEE Access, vol. 7, pp. 78665-78674, 2019.

[29] T. Hwu, A. Y. Wang, N. Oros, and J. L. Krichmar, “Adaptive Robot Path Planning Using a Spiking Neuron Algorithm With Axonal Delays," IEEE Trans. Cogn. Dev. Syst., vol. 10, no. 2, pp. 126-137, Jun. 2018.

[30] P. Sun and Z. Yu, "Tracking control for a cushion robot based on fuzzy path planning with safe angular velocity," IEEE/CAA J. Autom. Sin., vol. 4, no. 4, pp. 610-619, 2017.

[31] L. Liu, J. Yao, D. He, J. Chen, J. Huang, H. Xu, B. Wang, and J. Guo "Global Dynamic Path Planning Fusion Algorithm Combining JumpA* Algorithm and Dynamic Window Approach," IEEE Access, vol. 9, pp. 19632-19638, 2021

[32] Z. XU, "Rotary unmanned aerial vehicles path planning in rough terrain based on multi-objective particle swarm optimization,” J. Syst. Eng. Electron., vol. 31, no. 1, pp. 130-141, Jan. 2020

[33] J. Yuan, H. Wang, C. Lin, D. Liu, and D. Yu, "A Novel GRU-RNN Network Model for Dynamic Path Planning of Mobile Robot," IEEE Access, vol. 7, pp. 15140-15151, 2019.

[34] D. Kularatne, S. Bhattacharya, and M. A. Hsieh, "Optimal Path Planning in Time-Varying Flows Using Adaptive Discretization," IEEE Robot. Autom. Lett., vol. 3, no. 1, pp. 458-465, Jan. 2018.

[35] A. Ajam, A. A. Aziz, V. S. Asirvadam, A. S. Muda, I. Faye, and S. J. Safdar Gardezi, "A Review on Segmentation and Modeling of Cerebral Vasculature for Surgical Planning," IEEE Access, vol. 5, pp. 15222-15240, 2017

[36] S. Sun, T. Endo, and F. Matsuno, "Iterative Learning Control Based Robust Distributed Algorithm for Non-Holonomic Mobile Robots Formation,” IEEE Access, vol. 6, pp. 61904-61917, 2018.

[37] S.-Y. Chen and M.-H. Song, "Energy-Saving Dynamic Bias Current Control of Active Magnetic Bearing Positioning System Using Adaptive Differential Evolution," IEEE Trans. Syst. Man, Cybern. Syst., vol. 49, no. 5, pp. 942-953, May 2019.

[38] A. Francis, A. Faust, H.-T. L. Chiang, J. Hsu, J. C. Kew, M. Fiser, and T.-W. E. Lee, "Long-Range Indoor Navigation With PRM-RL," IEEE Trans. Robot., vol. 36, no. 4, pp. 1115-1134, Aug. 2020.

[39] G. Chong, H. Ramiah, J. Yin, J. Rajendran, P.-I. Mak, and R. P. Martins, "A Wide-PCE-Dynamic-Range CMOS Cross-Coupled Differential-Drive Rectifier for Ambient RF Energy Harvesting," IEEE Trans. Circuits Syst. II Express Briefs, vol. 68, no. 6, pp. 17431747, Jun. 2021

[40] D. Li, D. Fooladivanda, and S. Martinez, "Online Learning of Parameterized Uncertain Dynamical Environments with Finitesample Guarantees," in 2021 American Control Conference (ACC), 2021, vol. 2021-May, no. 4, pp. 2005-2010.

[41] Y. Maddahi and K. Zareinia, "Nonparametric Bootstrap Technique to Improve Positional Accuracy in Mobile Robots With Differential Drive Mechanism," IEEE Access, vol. 8, pp. 158502-158511, 2020.

[42] Q. Lou, F. Gonzalez, and J. Kovecses, "Kinematic Modeling and State Estimation of Exploration Rovers," IEEE Robot. Autom. Lett., vol. 4, no. 2, pp. 1311-1318, Apr. 2019

[43] F. Ke, Z. Li, and C. Yang, "Robust Tube-Based Predictive Control for Visual Servoing of Constrained Differential-Drive Mobile Robots," IEEE Trans. Ind. Electron., vol. 65, no. 4, pp. 3437-3446, Apr. 2018.

[44] C. Hu, R. Wang, F. Yan, Y. Huang, H. Wang, and C. Wei, "Differential Steering Based Yaw Stabilization Using ISMC for
Independently Actuated Electric Vehicles," IEEE Trans. Intell. Transp. Syst., vol. 19, no. 2, pp. 627-638, Feb. 2018.

[45] G. Chong, H. Ramiah, J. Yin, J. Rajendran, W. R. Wong, P.-I. Mak, and R. P. Martins, "CMOS Cross-Coupled Differential-Drive Rectifier in Subthreshold Operation for Ambient RF Energy Harvesting-Model and Analysis," IEEE Trans. Circuits Syst. II Express Briefs, vol. 66, no. 12, pp. 1942-1946, Dec. 2019.

[46] D. Chwa and J. Boo, “Adaptive Fuzzy Output Feedback Simultaneous Posture Stabilization and Tracking Control of Wheeled Mobile Robots With Kinematic and Dynamic Disturbances," IEEE Access, vol. 8, pp. 228863-228878, 2020.

[47] Y. Sun, C. Huang, Q. Zhang, Y. Liu, and L. Liang, "Analysis and Simulation of the Electrical-Differential System of a Grab-ShipUnloader-Crane," IEEE Access, vol. 9, pp. 8564-8580, 2021.

[48] L. Grasso, G. Sorbello, E. Ragonese, and G. Palmisano, "Codesign of Differential-Drive CMOS Rectifier and Inductively Coupled Antenna for RF Harvesting," IEEE Trans. Microw. Theory Tech., vol. 68, no. 1, pp. 365-376, Jan. 2020.

[49] J. Wang, Z. Luo, Y. Wang, B. Yang, and F. Assadian, "Coordination Control of Differential Drive Assist Steering and Vehicle Stability Control for Four-Wheel-Independent-Drive EV," IEEE Trans. Veh. Technol., vol. 67, no. 12, pp. 11453-11467, Dec. 2018.

[50] Q. Lu, Z. Li, L. Yu, and C.-Y. Su, "Adaptive Visual Regulation of Wheeled Mobile Robots: a Switching Approach," J. Intell. Robot. Syst., vol. 98, no. 2, pp. 345-358, May 2020.

[51] Q. Yao, Z. Zheng, L. Qi, H. Yuan, X. Guo, M. Zhao, Z. Liu, and T. Yang, "Path Planning Method With Improved Artificial Potential Field-A Reinforcement Learning Perspective," IEEE Access, vol. 8, pp. 135513-135523, 2020.

[52] J. Raj, K. Raghuwaiya, and J. Vanualailai, "Novel Lyapunov-Based Autonomous Controllers for Quadrotors," IEEE Access, vol. 8, pp. 47393-47406, 2020.

[53] D. Chen, X. Zhou, J. Li, J. He, X. Yu, L. Zhang, and W. Qi, “A Muscle Teleoperation System of a Robotic Rollator Based on Bilateral Shared Control," IEEE Access, vol. 8, pp. 151160-151170, 2020.

[54] S. Dixit, U. Montanaro, M. Dianati, D. Oxtoby, T. Mizutani, A. Mouzakitis, and S. Fallah, "Trajectory Planning for Autonomous High-Speed Overtaking in Structured Environments Using Robust MPC," IEEE Trans. Intell. Transp. Syst., vol. 21, no. 6, pp. 23102323, Jun. 2020

[55] B. Liu, W. Feng, T. Li, C. Hu, and J. Zhang, “A Variable-Step RRT * Path Planning Algorithm for Quadrotors in Below-Canopy," IEEE Access, vol. 8, pp. 62980-62989, 2020.

[56] D. Zhao, H. Yu, X. Fang, L. Tian, and P. Han, “A Path Planning Method Based on Multi- Objective Cauchy Mutation Cat Swarm Optimization Algorithm for Navigation System of Intelligent Patrol Car," IEEE Access, vol. 8, pp. 151788-151803, 2020.

[57] J. Huang, W. Sun, and Y. Gao, "A Method of Trajectory Planning for Unmanned Aerial Vehicle Formation Based on Fluid Dynamic Model," IEEE Access, vol. 8, pp. 2824-2834, 2020.

[58] A. C. Woods, H. M. Lay, and Q. P. Ha, "A novel extended potential field controller for use on aerial robots," in 2016 IEEE International Conference on Automation Science and Engineering (CASE), 2016, pp. 286-291.

[59] D. Jin, Z. Fang, and J. Zeng, "A Robust Autonomous following Method for Mobile Robots in Dynamic Environments," IEEE Access, vol. 8, pp. 150311-150325, 2020

[60] S. Park, H. T. Kim, and H. Kim, "VMCS: Elaborating APF-Based Swarm Intelligence for Mission-Oriented Multi-UV Control," IEEE Access, vol. 8, pp. 223101-223113, 2020.

[61] B. Li and Y. Wu, "Path Planning for UAV Ground Target Tracking via Deep Reinforcement Learning," IEEE Access, vol. 8, pp. 2906429074, 2020.

[62] Q. Yao, Z. Zheng, L. Qi, H. Yuan, X. Guo, M. Zhao, Z. Liu, and T. Yang, "Path Planning Method With Improved Artificial Potential Field-A Reinforcement Learning Perspective," IEEE Access, vol. 8, pp. 135513-135523, 2020 .

[63] Z. Yuan, Y. Yang, Y. Hu, and X. Ma, "Channel-Aware Potential Field Trajectory Planning for Solar-Powered Relay UAV in Near-Space," IEEE Access, vol. 8, pp. 143950-143961, 2020. 
[64] E. Wu, Y. Sun, J. Huang, C. Zhang, and Z. Li, "Multi UAV Cluster Control Method Based on Virtual Core in Improved Artificial Potential Field," IEEE Access, vol. 8, pp. 131647-131661, 2020.

[65] A. A. Ravankar, A. Ravankar, T. Emaru, and Y. Kobayashi, "HPPRM: Hybrid Potential Based Probabilistic Roadmap Algorithm for Improved Dynamic Path Planning of Mobile Robots," IEEE Access, vol. 8, pp. 221743-221766, 2020.

[66] O. Khatib, "Real time obstacle avoidance for manipulators and mobile robots," International Journal of Robotics and Research, vol. 5, no. 1. pp. 90-98, 1986.
[67] B. Lu, G. Li, H. Yu, H. Wang, J. Guo, D. Cao, and H. He, “Adaptive Potential Field-Based Path Planning for Complex Autonomous Driving Scenarios,” IEEE Access, vol. 8, pp. 225294-225305, 2020.

[68] A. Lazarowska, "Comparison of Discrete Artificial Potential Field Algorithm and Wave-Front Algorithm for Autonomous Ship Trajectory Planning," IEEE Access, vol. 8, pp. 221013-221026, 2020. 